\title{
Caffeine and nicotine adsorption on perfect, defective and porous
}

\section{graphene sheets}

\author{
R. Majidi ${ }^{*}, 1$ and A.R. Karami ${ }^{2}$ \\ ${ }^{1}$ Department of Physics, Shahid Rajaee Teacher Training University, Lavizan, 16788-15811 Tehran, Iran \\ ${ }^{2}$ Department of Chemistry, Shahid Rajaee Teacher Training University, Lavizan, 16788-15811 Tehran, Iran
}

\begin{abstract}
Adsorption of caffeine and nicotine molecules on perfect, defective and porous graphene sheets is investigated using density functional theory. The high adsorption energies indicate strong adsorption of caffeine and nicotine molecules on graphene sheets. It was found that caffeine adsorption is stronger in comparison to nicotine adsorption. The electronic band structures analysis shows that the electronic properties of graphene sheets are sensitive to the presence of caffeine and nicotine molecules. The graphene sheets become n-type semiconductors with caffeine and nicotine adsorption. Our results suggest the possibility of using graphene based sensors for caffeine and nicotine detection.
\end{abstract}

Keywords: Density functional theory; Graphene; Stone Wales defect; Porous; Sensor.

\section{Introduction}

Caffeine and nicotine are both alkaloids and therapeutic compounds [1, 2]. Tea, coffee, cocoa beans, guarana and yerba mate contain naturally caffeine [3]. Cigarettes, cigars, 
snuff, and pipe tobacco are common sources of nicotine. Furthermore, caffeine and nicotine are found in the stimulant family of drugs [3]. These drugs carry a number of side effects and overdose of them causes serious problems. In particular, caffeine and nicotine overdose can cause insomnia, withdrawal symptoms, rapid breathing, heart palpitations and high blood pressure [3]. Therefore, fabrication of sensitive sensors and filters with high sorption capacity is essential for detection of caffeine and nicotine molecules.

The recent advances in nanotechnology have enabled researchers to design and fabricate sensors using nanostructures. Many sensors based on graphene, carbon nanotubes, graphyne sheets and tubes have been suggested for detection of different molecules [4-18]. For instance, possibility of using perfect and defective carbon nanotubes for caffeine and nicotine detection was reported [12, 13]. It was shown that carbon nanotubes can be used as caffeine and nicotine sensors due to sensitivity of their electronic properties to the presence of these molecules [12, 13]. The good sensor properties of carbon nanotubes are already known for some time [14], but recently, gas sensing behavior of graphene was also reported [15]. It was found that graphene is proper for detecting individual molecules, adsorbed from gas phase or even larger molecules that would be attached to a graphene sheet from solution, indicating its applicability in sensing wide rage of molecules or even biomolecules [16-19]. These excellent sensor applications of graphene are due to its two dimensional structure which maximizes the effect of surface dopants and its special electronic properties [18]. The present results motivated us to investigate possibility of using graphene for caffeine and nicotine detection in the present work.

In reality, graphene usually suffers from various types of defect in the atomic structure during its growth [20]. It was shown that defects such as Stone Wales (SW) modify the electronic properties and chemical reactivity of graphene [21, 22]. 
Comparison the adsorption mechanism of different molecules on perfect and defective graphene sheets indicates that SW defect can make the adsorption stronger. In addition, it was found that the defective graphene is more sensitive to the presence of molecules than the perfect graphene [23, 24].

The use of graphene in electronic applications is nowadays hindered by the fact that graphene is a gap less semiconductor. To solve this problem, many other twodimensional structures with non-zero band gap have become objects of interest. Fortunately, recent advances have made possible the synthesis of intrinsically hydrogenated structures such as porous graphene [25]. The porous graphene sheets have the advantage of retaining some properties of graphene while being intrinsically non-zero gap structure $[25,26]$. Hence, this kind of carbon based nanostructures opens new perspectives to novel applications and will bring more surprise in the future [27]. In the present study, we have carried out the possibility of using perfect, defective and porous graphene for caffeine and nicotine detection by using density functional theory (DFT).

\section{Computational details}

The DFT calculations are performed by using OpenMX3.7 package [28]. The generalized gradient approximation (GGA) with Perdew-Burke-Ernzerhof (PBE) correction is considered for the exchange-correlation function [29]. The van der Waals interactions between molecules and sheets are described with Grimme’s method [30]. The plane wave basis set is restricted by a cutoff energy of 150 Ry. A cutoff radius of 6 bohr is chosen for all basis functions, namely, C6.0-s2p2d1, O6.0-s2p2d1, N6.0-s2p2d1 and H6.0-s1p1. All structures are optimized until the forces acting on relaxed atoms are smaller than $0.01 \mathrm{eV} / \AA$. The K-point is set to be 41 along each high symmetry lines in 
the Brillouin zone. The charge transfer that occurs in the system is measured by using the Mulliken charge analysis.

The atomic structures of caffeine and nicotine molecules are shown in Fig. 1. The perfect, defective and porous graphene sheets are selected as the host materials for adsorption of caffeine and nicotine molecules. We have considered supercells of perfect and defective graphene sheets with $9 \times 9$ unit cells and supercell of porous graphene sheet with $3 \times 3$ unit cells. These supercells and the optimized bond lengths are presented in Fig. 2. As shown in Fig. 2(b), one SW defect is considered in one supercell to construct defective graphene sheet. The periodic boundary conditions are applied to the supercells. The simulation boxes are assumed $22.15 \times 22.15 \times 20 \AA^{3}$. A vacuum width of about $20 \AA$ is considered to ensure there is negligible interaction between graphene sheets in neighboring cells.

To evaluate stability of adsorbed molecules on graphene sheets, adsorption energy is calculated as

$E_{\text {ads }}=E_{\text {molecule }+ \text { sheet }}-E_{\text {sheet }}-E_{\text {molecule }}$

where $E_{\text {molecule+sheet }}$ is the total energy of graphene sheet with adsorbed molecule, $E_{\text {sheet }}$ is the total energy of graphene sheet and $E_{\text {molecule }}$ is the total energy of an isolated molecule.

\section{Results and discussions}

The most stable adsorption configurations of caffeine and nicotine molecules on graphene sheets are investigated by locating the molecules with various orientations on different possible adsorption sites of the sheets. Upon the lowest adsorption energies, the most stable adsorption configurations for caffeine and nicotine molecules on graphene sheets are identified. From our calculations, we found that the molecules are preferred to orient in parallel with respect to the graphene sheets. The energetically 
most favorable adsorption sites are shown with label $\times$ on graphene sheets in Fig. 2. It means, the center of the hexagonal ring of molecule (labeled by * in Fig. 1) is placed above the positions (labeled by $\times$ in Fig. 2 ) in the most stable configurations. The most stable adsorption configurations of caffeine and nicotine adsorbed on graphene sheets are shown in Fig. 3. After determining the adsorption sites, the molecules are vertically moved toward and away from the sheets to determine the optimal adsorption distance. We have defined the distance between $\times$ and $*$ labels as the adsorption distance (Fig. 3). The optimal adsorption distances and adsorption energies of the most stable configurations are summarized in Table 1. The high adsorption energies indicate chemisorption of caffeine and nicotine molecules on graphene sheets. Adsorption on perfect graphene sheet is stronger than on defective and porous graphene sheets. Adsorption of caffeine is stronger than adsorption of nicotine.

We have compared the electronic band structures of graphene sheets before and after caffeine and nicotine adsorption to understand the effect of molecules on the electronic properties of graphene sheets. The electronic band structure of perfect graphene is shown in Fig. 4(a). As expected, the valence and conduction bands cross linearly each other and perfect graphene is a semimetal. The electronic band structures of perfect graphene after caffeine and nicotine adsorption are shown in Fig. 4(b) and Fig. 4(c), respectively. Here, adsorption of molecules leads to appear of small band gap and occupied states below the Fermi level. It means perfect graphene become n-type semiconductor with caffeine and nicotine adsorption. The energy band gaps between the highest occupied and lowest unoccupied states of graphene before and after adsorption of molecules are compared in Table 2.

The electronic band structure of graphene with SW defect is shown in Fig. 5(a). It is clear that the presence of SW defect opens a small band gap of $0.04 \mathrm{eV}$. Hence, graphene with SW defect is a semiconductor in good agreement with previous studies 
[31, 32]. Adsorption of caffeine and nicotine molecules on defective graphene leads to appear occupied states below the Fermi level (Fig. 5(b) and Fig. 5(c)). This result indicates defective graphene becomes n-type semiconductor in the presence of caffeine and nicotine molecules. The energy band gaps are listed in Table 2.

The electronic band structures of porous graphene before and after caffeine and nicotine adsorption are presented in Fig. 6. As shown in Fig. 6(a), porous graphene is an insulator with a large band gap of $2.12 \mathrm{eV}$. This result is in good agreement with previous studies which report band gap of 3.3 [26], 2.35 [33] and $2.22 \mathrm{eV}$ [34]. As shown in Fig. 6(b) and Fig. 6(c), adsorption of caffeine and nicotine molecules leads to occur occupied states below the Fermi level in the forbidden band. Hence, porous graphene become n-type semiconductor with caffeine and nicotine adsorption. In Table 2, the energy band gaps of porous graphene before and after adsorption of nicotine and caffeine are presented. The change in band gap of porous graphene caused by molecular adsorption is larger than perfect and defective sheets. The highly sensitivity of the electronic properties of porous graphene sheet indicate that porous graphene is more suitable for sensor applications than other types of graphene sheets.

The Mulliken population analysis clarifies that electrons are transferred from caffeine and nicotine molecules to graphene sheets. The amount of charge transfer between adsorbed molecules and graphene sheets is presented in Table 1 . The results indicate caffeine and nicotine molecules act as electron donors. Adsorption of these donor molecules increase the number of electrons in graphene sheets and thus enhance the electrical conductivity of graphene sheets. Here, the n-type semiconducting properties of graphene sheets are attributed to adsorption of these donor molecules.

\section{Conclusions}


The interaction of caffeine and nicotine molecules with perfect, defective and porous graphene sheets is studied using DFT. A detailed investigation is performed on the adsorption energy, adsorption structure, charge transfer and electronic band structure. It is shown that caffeine and nicotine molecules are strongly chemisorbed on perfect, defective and porous graphene sheets. For perfect graphene, the binding strength of these molecules is found to be stronger than defective and porous graphene. The caffeine presents more strong interaction with graphene sheets rather than nicotine. The perfect, defective and porous graphene sheets become n-type semiconductors with adsorption of charge donor molecules such as caffeine and nicotine. The sensitivity of the electronic properties of graphene sheets to the presence of caffeine and nicotine indicate graphene sheets are promising nanostructures for detection of these molecules. It is also found that porous graphene sheet is more suitable to the sensor material because its change of band gap caused by caffeine and nicotine adsorption is larger than other types of graphene sheets.

\section{Acknowledgment}

The work is supported by Iran National Science Foundation: INSF. 


\section{References}

[1] J. Rusted, L. Graupner, N. O’Connell, C. Nicholls, Does nicotine improve cognitive function? Psychopharmacology 115 (1994) 547.

[2] Y. Xu, J.H. Zhu, L.L. Ma, J.A. An, Y.L. Wei, X.Y. Shang, Removing nitrosamines from mainstream smoke of cigarettes by zeolites, Microporous and Mesoporous Mater. 60 (2003) 125.

[3] http://www.livestrong.com/article/91383-caffeine-nicotine/

[4] J. Zhao, A. Buldum, J. Han, J.P. Lu, Gas molecule adsorption in carbon nanotubes and nanotube bundles, Nanotechnology 13 (2002) 195.

[5] M. Chi, Y.P. Zhao, First principle study of the interaction and charge transfer between graphene and organic molecules, Comput. Mater. Sci. 56 (2012) 79.

[6] M. Chi, Y.P. Zhao, Adsorption of formaldehyde molecule on the intrinsic and Aldoped graphene: A first principle study, Comput. Mater. Sci. 46 (2009) 1085.

[7] R. Majidi, A biosensor for hydrogen peroxide detection based on electronic properties of carbon nanotubes, Mol. Phys. 111 (2012) 89.

[8] E. Llobet, Gas sensors using carbon nanomaterials: A review, Sens. Act. B: Chemical 179 (2013) 32.

[9] R. Majidi, A.R. Karami, Nitrotyrosine adsorption on carbon nanotube: A density functional theory study, Indian J. Phys. 88 (2014) 483.

[10] A.R. Karami, R. Majidi, Detection of toxic gases with graphyne Nanotubes, A density functional theory study, Chem. Lett. 44 (2015) 1071.

[11] R. Majidi, A.R. Karami, Adsorption of formaldehyde on graphene and graphyne, Physica E 59 (2014) 169.

[12] E.C. Girao, S.B. Fagan, I. Zanella, A.G.S. Filho, Nicotine adsorption on single wall carbon nanotubes, J. Hazard. Mater. 184 (2010) 678. 
[13] H.J. Lee, G. Kim, Y.K. Kwon, Molecular adsorption study of nicotine and caffeine on single-walled carbon nanotubes from first principles Chem. Phys. Lett. 580 (2013) 57.

[14] J. Kong, N.R. Franklin, C. Zhou, M.G. Chapline, S. Peng, K. Cho, H. Dai, Nanotube molecular wires as chemical sensors, Science 287 (2000) 622.

[15] F. Schedin, A.K. Geim, S.V. Morozov, E.W. Hill, P. Blake, M.I. Katsnelson, K.S. Nocoselov, Detection of individual gas molecules adsorbed on graphene, Nat. Mat. 6 (2007) 652.

[16] M. Zhang, C. Liao, C.H. Mak, F. Yan, Highly sensitive glucose sensors based on enzyme-modified whole-graphene solution-gated transistors, Sci. Rep. 5 (2015) 1.

[17] B.N. Papas, I.D. Petsalakis, G. Theodorakopoulos, J.L. Whitten, CI and DFT studies of the adsorption of the nerve agent sarin on surfaces, J. Phys. Chem. C 118 (2014) 23042.

[18] O. Leenaerts, B. Partoens, F. M. Peeters, Adsorption of $\mathrm{H}_{2} \mathrm{O}, \mathrm{NH}_{3}, \mathrm{CO}, \mathrm{NO}_{2}$, and NO on graphene: A first-principles study, Phys. Rev. B 77 (2008) 125416.

[19] N.K. Jaiswal, G. Kovacevic, B. Pivac, Reconstructed graphene nanoribbon as a sensor for nitrogen based molecules, Appl. Surf. Sci. 357 (2015) 55.

[20] J. Ma, D. Alfe, A. Michaelides, E. Wang, Stone-Wales defects in graphene and other planar sp2-bonded materials, Phys. Rev. B 80 (2009) 033407.

[21] Q. Zhou, C. Wang, Z. Fu, Y. Tang, H. Zhang, Adsorption of formaldehyde molecule on Stone-Wales defected graphene doped with Cr, Mn, and Co: A theoretical study, Comput. Mater. Sci. 83 (2014) 398.

[22] Q. Zhou, Y. Tang, C. Wang, Z. Fu, H. Zhang, Electronic and magnetic properties of transition-metal atoms absorbed on Stone-Wales defected graphene sheet: A theory study, Comput. Mater. Sci. 81 (2014) 348. 
[23] B. Akdim, T. Kar, X. Duan, R. Pachter, Density functional theory calculations of ozone adsorption on sidewall single-wall carbon nanotubes with Stone-Wales defects, Chem. Phys. Lett. 445 (2007) 281.

[24] X. Qin, Q. Meng, W. Zhao, Effects of Stone-Wales defect upon adsorption of formaldehyde on graphene sheet with or without Al dopant: A first principle study, Surf. Sci. 605 (2011) 930.

[25] D.E. Jiang, V.R. Cooper, Z.F. Chen, Porous graphene as the ultimate membrane for gass, Nano. Lett. 9 (2009) 4019.

[26] G. Brunetto, P.A.S. Autreto, L.D. Machado, B.I. Santos, R.P.B. dos Santos, D.S. Galvao, Nonzero gap two-dimensional carbon allotrope from porous graphene, J. Phys. Chem. C 116 (2012) 12810.

[27] X. Pengtao, Y. Jixiang, W. Kesai, Z. Zhen, S. Panwen, Porous graphene: Properties, preparation, and potential applications, Chin. Sci. Bull. 57 (2012) 2948.

[28] T. Ozaki, H. Kino, J. Yu, M.J. Han, N. Kobayashi, M. Ohfuti, F. Ishii, et al. User’s manual of OpenMX version 3.7. <http://www.openmx-square.org>.

[29] J.P. Perdew, K. Burke and M. Ernzerhof, Generalized gradient approximation made simple, Phys. Rev. Lett. 77 (1996) 3865.

[30] S. Grimme, Semiempirical GGA-type density functional constructed with a longrange dispersion correction, J. Comput. Chem. 27 (2006) 1787.

[31] L. Chen, H. Hu, Y. Ouyang, H.Z. Pan, Y.Y. Sun, F. Liu, Atomic chemisorption on graphene with Stone-Thrower-Wales defects, Carbon 49 (2011) 3356.

[32] R. Majidi, A.R. Karami, Nitrotyrosine adsorption on defective graphene: A density functional theory study, Physica E 70 (2015) 170.

[33] Y. Ding, Y. Wang, S. Shi and W. Tang, Electronic structures of porous graphene, $\mathrm{BN}$, and $\mathrm{BC}_{2} \mathrm{~N}$ sheets with one- and two-hydrogen passivations from first principles, $\mathrm{J}$. Phys. Chem. C 115 (2011) 5334. 
[34] X. Liang, Y.S. Jung, S. Wu, A. Ismach, D.L. Olynick, S. Cabrini and J. Bokor, Formation of band gap and subbands in graphene nanomeshes with Sub-10 nm ribbon width fabricated via nanoimprint lithography, J. Nano. Lett. 10 (2010) 2454. 


\section{Table caption}

Table 1 The optimal adsorption distance, $d_{\times-*}$, adsorption energy, $E_{\text {ads, }}$, and charge transfer, $q$.

Table 2 The energy band gap (eV) before and after adsorption of caffeine and nicotine. 
Table 1 The optimal adsorption distance, $d_{\times-*}$, adsorption energy, $E_{\text {ads }}$ and charge transfer, $q$.

\begin{tabular}{|c|c|c|c|c|}
\hline \multirow{2}{*}{ perfect graphene } & & $d_{\times-*}(\AA)$ & $E_{\text {ads }}(\mathrm{eV})$ & $q(\mathrm{e})$ \\
\cline { 2 - 5 } & nicotine & 3.2 & -4.5 & 1.7 \\
\hline \multirow{2}{*}{ defective graphene } & caffeine & 2.5 & -8.1 & 2.1 \\
\cline { 2 - 5 } & nicotine & 3.4 & -4.3 & 1.4 \\
\hline \multirow{2}{*}{ porous graphene } & caffeine & 2.5 & -4.2 & 0.9 \\
\cline { 2 - 5 } & nicotine & 3.4 & -2.5 & 0.8 \\
\hline
\end{tabular}


Table 2 The energy band gap (eV) before and after adsorption of nicotine and caffeine.

\begin{tabular}{|c|c|c|c|}
\hline & no molecule & caffeine & nicotine \\
\hline perfect graphene & 0.00 & 0.02 & 0.02 \\
\hline defective graphene & 0.04 & 0.05 & 0.03 \\
\hline porous graphene & 2.12 & 0.97 & 0.14 \\
\hline
\end{tabular}




\section{Figure captions}

Fig. 1 Atomic structures of caffeine and nicotine molecules.

Fig. 2 Structures of perfect, defective and porous graphene sheets. Bond lengths are in Å.

Fig. 3 The most stable adsorption configurations of caffeine and nicotine adsorbed on (a-b) perfect, (c-d) defective and (e-f) porous graphene.

Fig. 4 Electronic band structures of (a) perfect graphene, (b) perfect graphene with caffeine and (c) perfect graphene with nicotine. (The Fermi level is at $0 \mathrm{eV}$.)

Fig. 5 Electronic band structures of (a) defective graphene, (b) defective graphene with caffeine and (c) defective graphene with nicotine. (The Fermi level is at $0 \mathrm{eV}$.)

Fig. 6 Electronic band structures of (a) porous graphene, (b) porous graphene with caffeine and (c) porous graphene with nicotine. (The Fermi level is at $0 \mathrm{eV}$.) 
Fig. 1

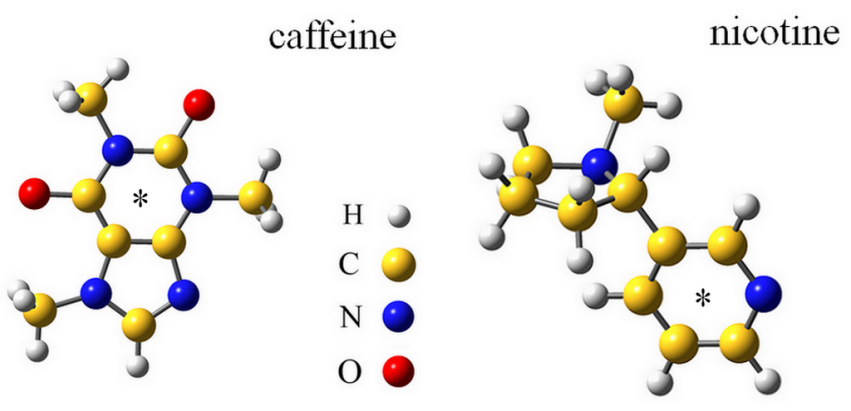


Fig. 2

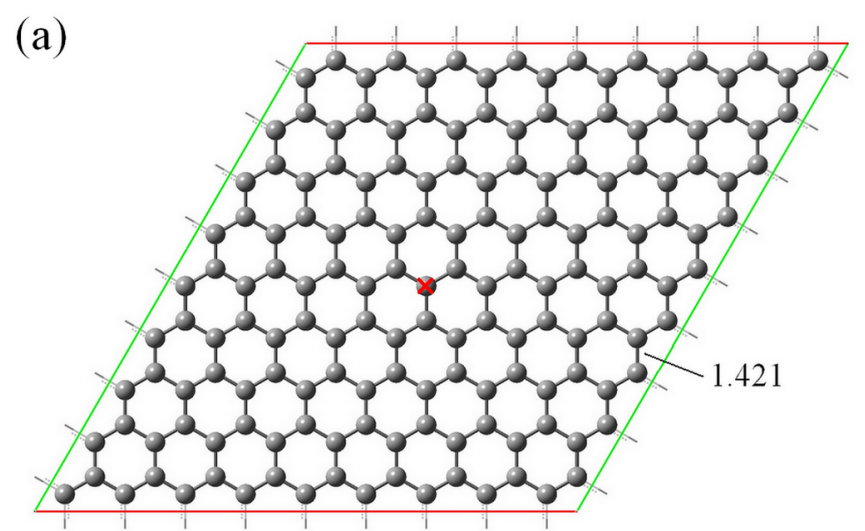

(b)

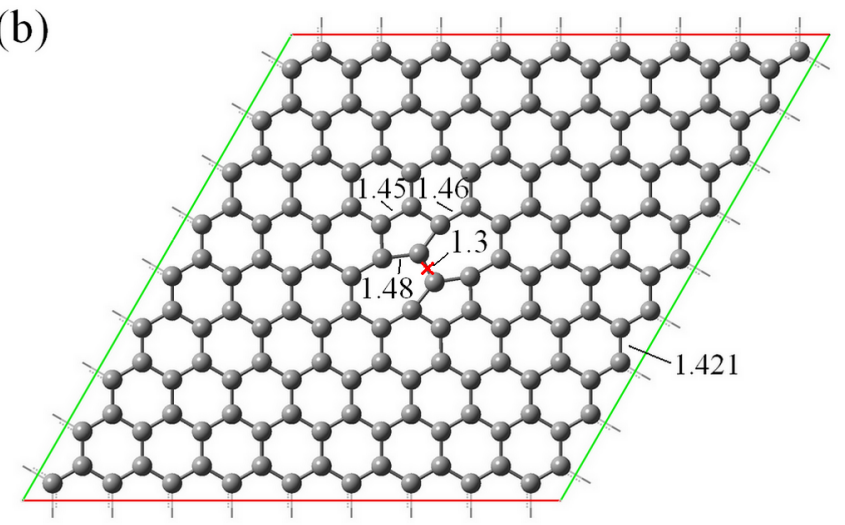

(c) 
Fig. 3
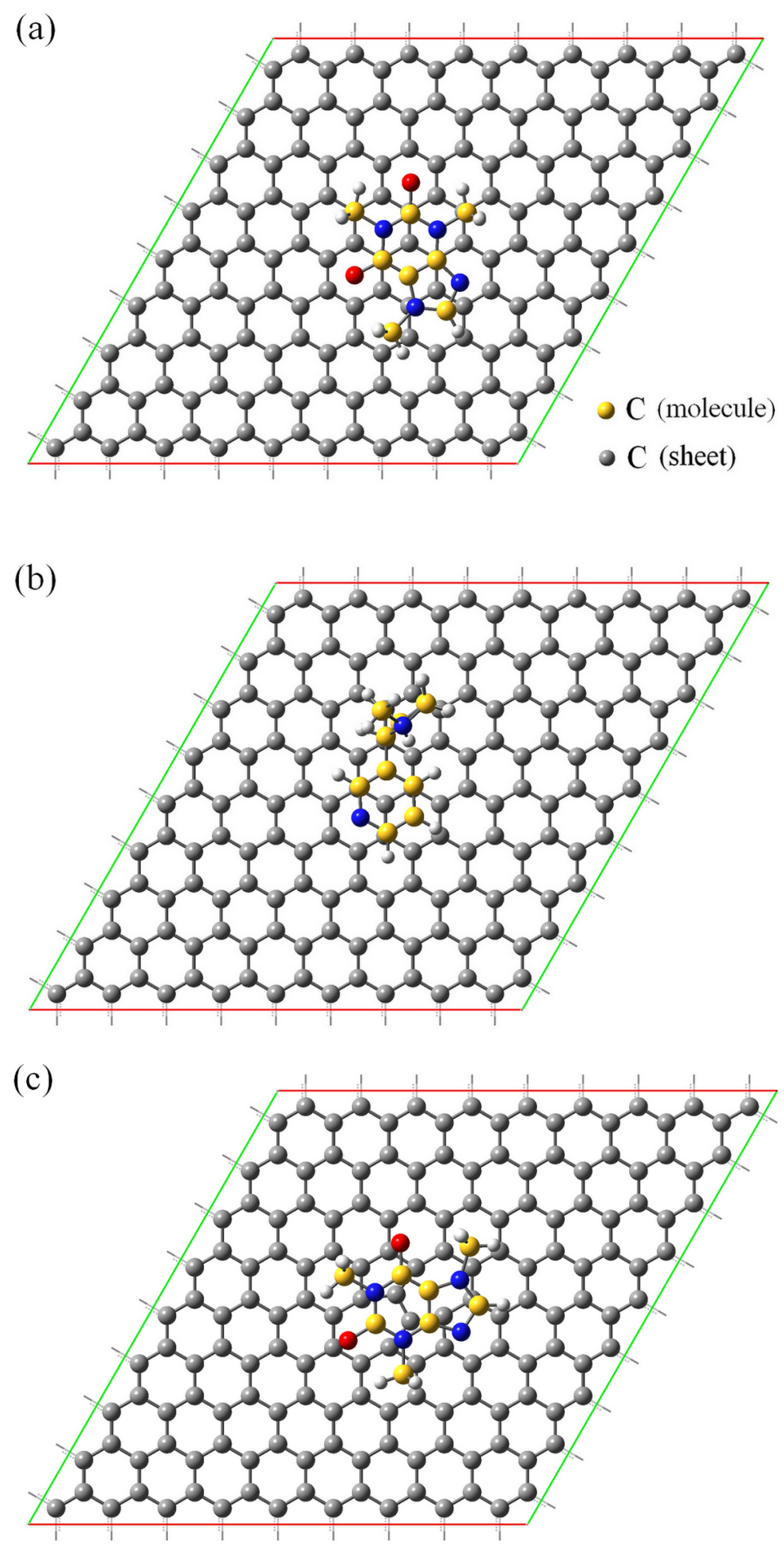

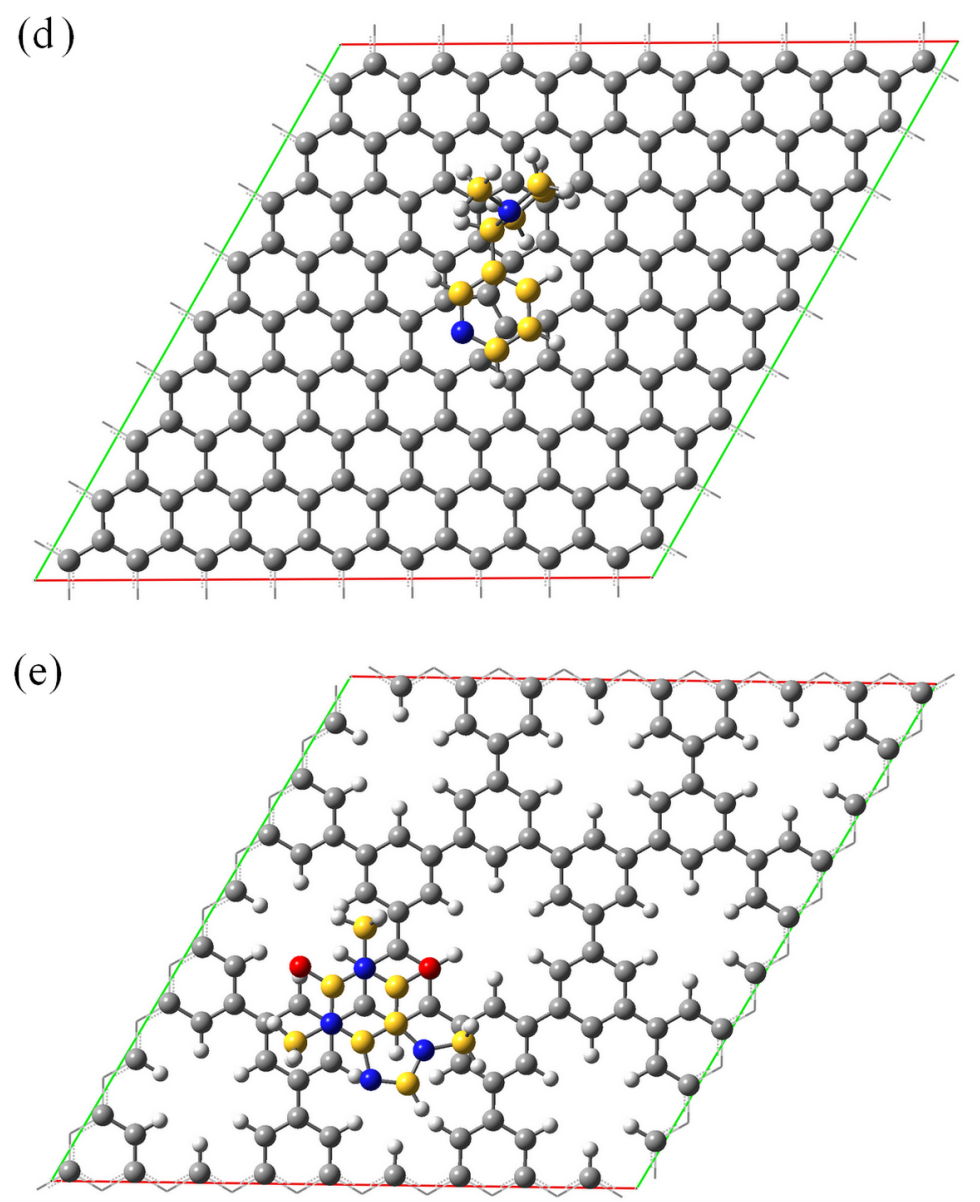

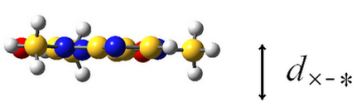

- - opug

(f)

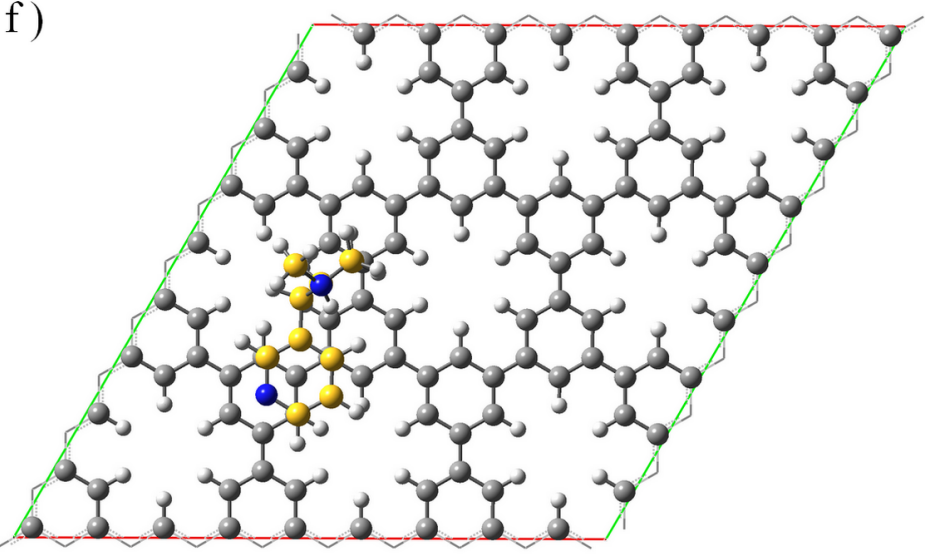

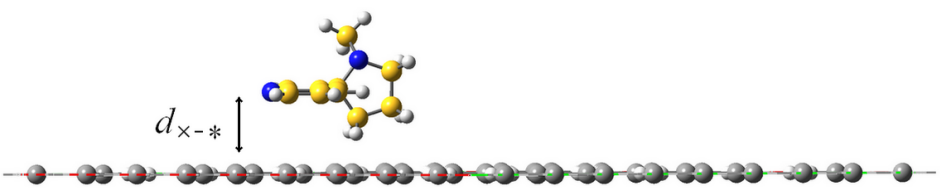


Fig. 4
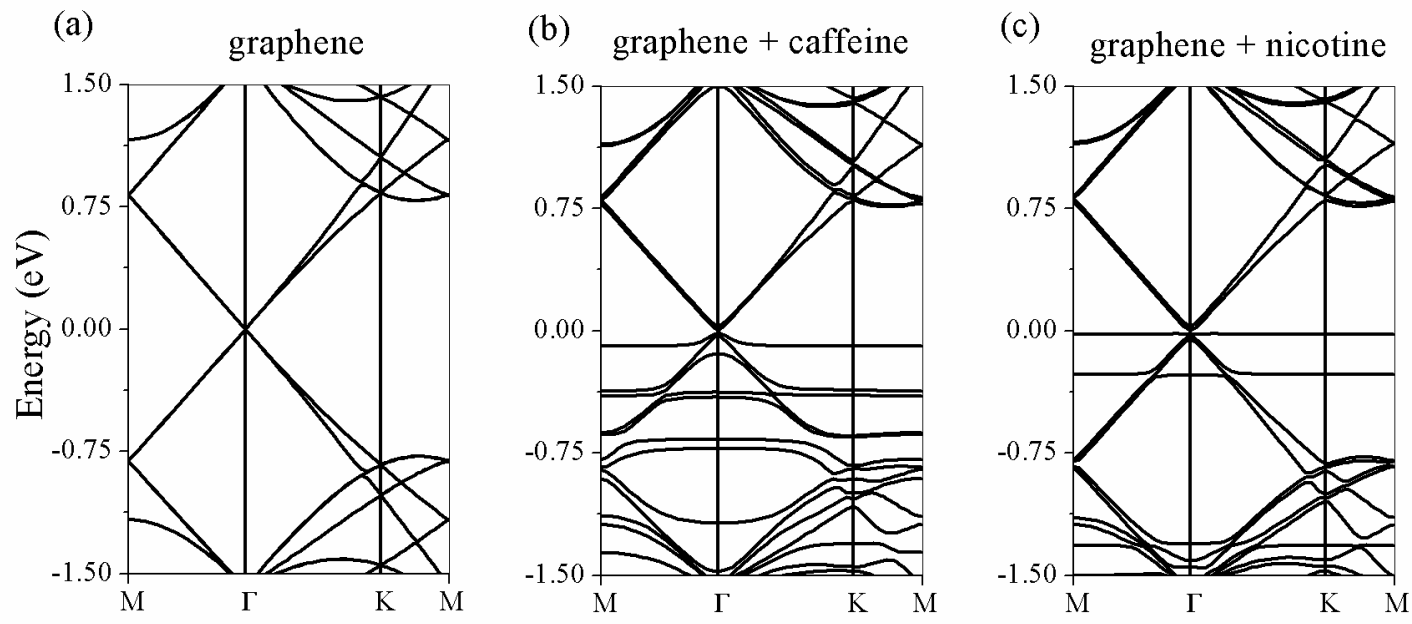
Fig. 5
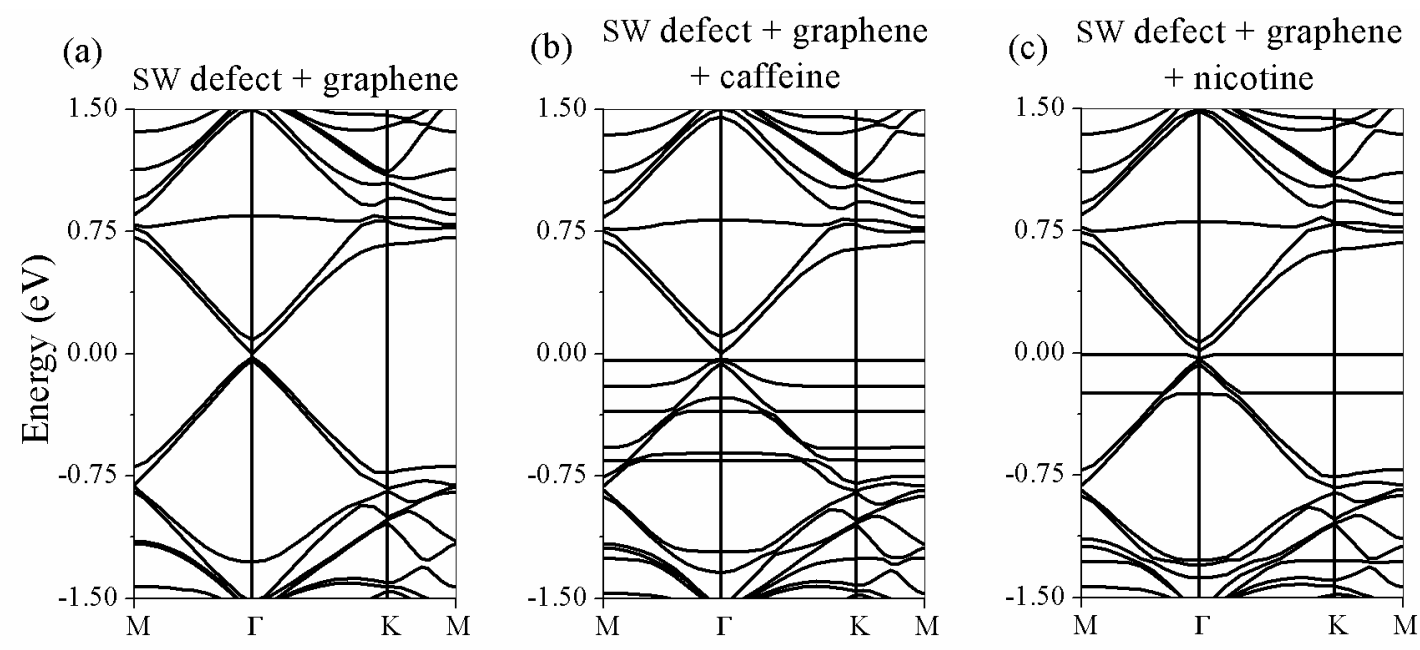
Fig. 6
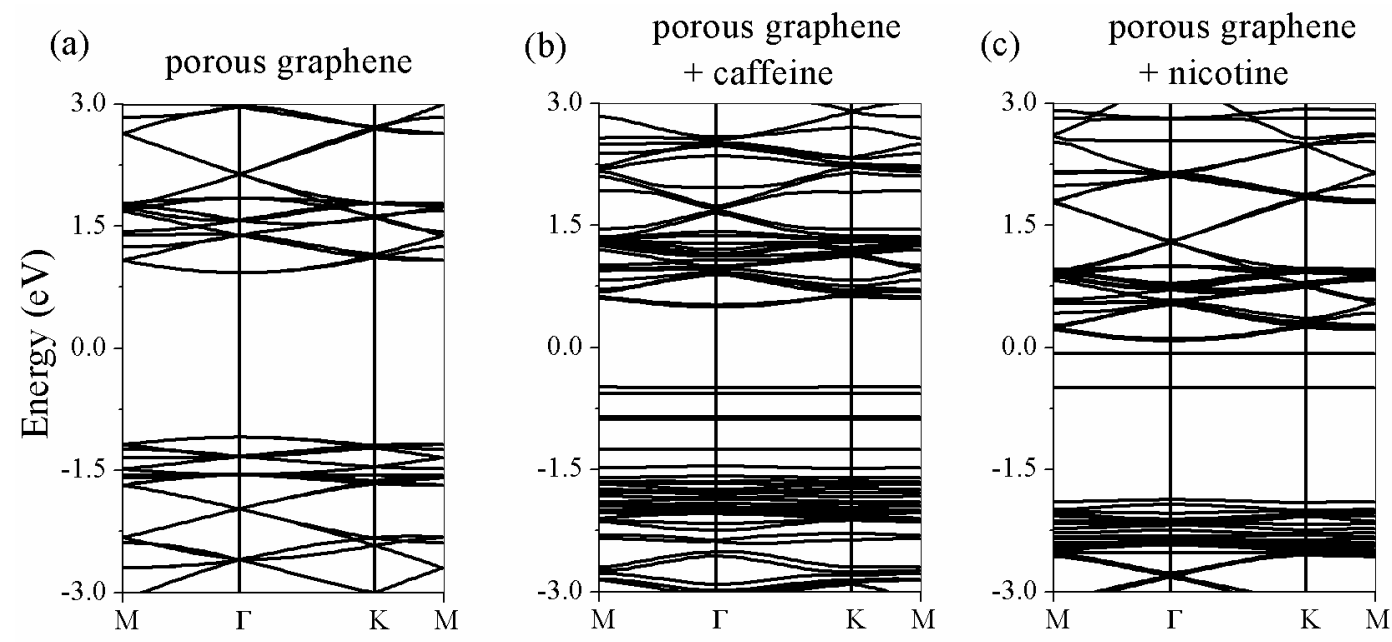